\title{
IOT-based Seat Belts Intelligent Monitoring System
}

\author{
Yeqiang Lu ${ }^{1, a}$, Hongyan Chen ${ }^{2, b}$ and Bing Zhang ${ }^{2, c}$ \\ ${ }^{1}$ Hangzhou Vocational and Technical College, Hangzhou 310000, China; \\ ${ }^{2}$ China Jiliang University, Hangzhou 310000, China. \\ a13858112568@163.com, bbbchy@163.com, czhangbingapple@qq.com
}

Keywords: Seat belts of passenger car, IOT, SBRD.

\begin{abstract}
Seat Belt Reminder Device as one of effective way to improve the rates of belt wearing, which be installed in every coach, but the SBRD only reminders the driver and the front passenger, while ignoring the rear passengers. The annual passenger vehicle accident reports show that there are always some injured passengers who do not wear seatbelts. Therefore, in order to improve the wearing rates of passenger car's seatbelt and reduce injuries due to accidents which are caused by not wearing seatbelts. In this paper, it is proposed Passenger Train Seat Belt Monitoring System Based on Internet of Things.
\end{abstract}

\section{Introduction}

Passenger transport vehicles as an important way of travel, the safety of passengers has been the problem of passenger transport companies and traffic management departments to consider. With the increase of vehicle ownership in our country, the speed of the vehicle is increased, and the traffic accident is increasing [1].

At present, the main safety belt on the existing passenger vehicles to remind the main test is the seat of the seat belt to wear the seat of the driver, and the seat belt for the seat of the seat belt wear testing has not been very good use. In this way, it will increase the number of people who are not used to wearing a seat belt of luck, to a certain extent, will affect the safety of their journey. Through the early field survey found that the passenger transport center for passenger vehicles on the wearing of seat belts, mainly rely on the driver's reminder and check the link when the check. However, this traditional inspection methods have many shortcomings, such as some travelers in order to cope with the inspection, and not really put the safety belt into the card slot, or after the removal of the safety belt $[2,3]$. These acts are sufficient to cause an accident when a passenger vehicle has a traffic accident, because it is not protected by a safety belt. We can from the traffic control department released over the years the passenger vehicle accident warning video see, whenever there is a traffic accident, some of the passengers because there is no safety belt is strong inertia instantly thrown from their seats, cause secondary damage. Therefore, in order to solve the above problems, it is necessary to develop a set of monitoring system, which is based on the Internet of things technology.

\section{The Overall Design}

The overall architecture of the system is shown in Fig. 1 System uses the traditional C/S architecture model. The client is composed of each vehicle cab terminal, the server is located in the terminal of the passenger terminal, and the terminal PC is also provided with a monitoring operation terminal. Vehicle with the passenger transportation center of remote communication and data transmission through Internet, cab of vehicle monitoring terminal is connected to the Internet via GPRS, and every seat is to communicate through the wireless chip and cab monitoring terminal. The whole system adopts three levels of structure, the idea of the Internet of things is applied to the system to solve the problem of remote monitoring, at the same time, it can monitor the status of each seat belt [2]. 


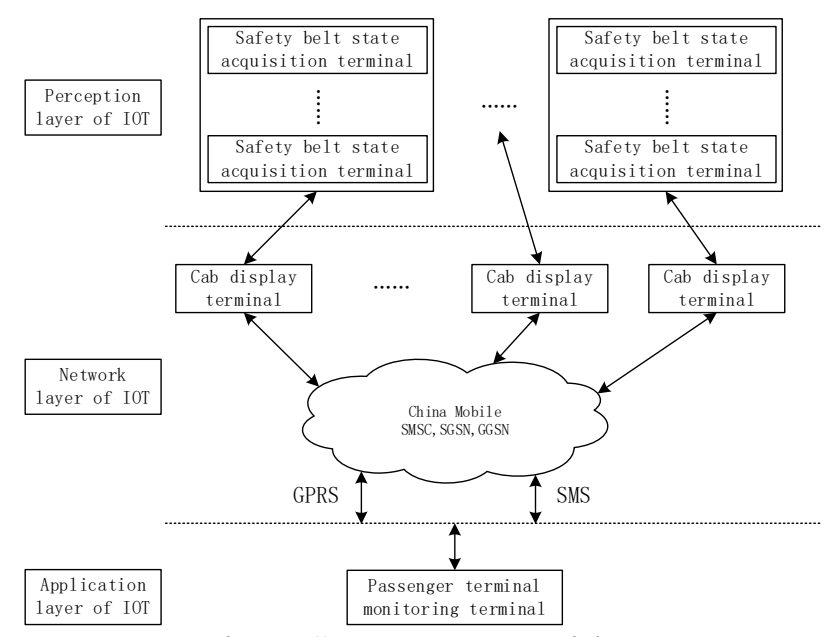

Fig. 1 System composition

The system is based on the Internet of things, the characteristics of the Internet of things is composed of multi sensor nodes, the sensor node of the system is all the seats inside the passenger car. For the combination of so many sensors, the Internet of things technology has a related processing mode, that is, wireless sensor network technology. Wireless sensor network is one of the main technologies of Internet of things, and it is a special kind of wireless communication network. There are three kinds of transmission models in Wireless Sensor Networks: data transfer model, Ad-hoc data transfer model and mobile sink model. Because of the fixed nodes, the base station is fixed, and the distance between them is fixed, all adopt the classical clustering data transfer model [3]. As shown in Fig. 2.

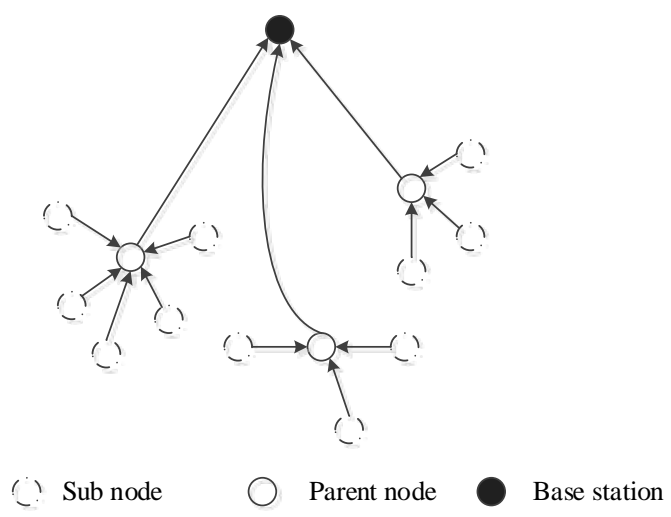

Fig. 2 Data transmission model

\section{System Design}

The hardware of this system is to realize the data collection, data transmission and data display of the safety belt in passenger car, and it is the front end of the whole monitoring system. Passenger car centralized controller, on the one hand, the acquisition to the passenger car of all seats seat and safety belt wear a timely sent to the monitoring center of passenger and receive passenger monitoring center reminder messages and inquiry instruction, so as to ensure the wearing of the real-time control and control monitoring center of each a transport vehicle in the safety belt.

As shown in Fig. 3, the design of passenger vehicle centralized controller based on embedded real-time operating system as a platform that integrates packet switching network module, a seat sensor nodes, sensor nodes and wireless transceiver transparent module buckle. The wireless transceiver module and the controller are connected by the SPI interface, and the packet switching network module and the controller communicate with the controller through the UART interface. 


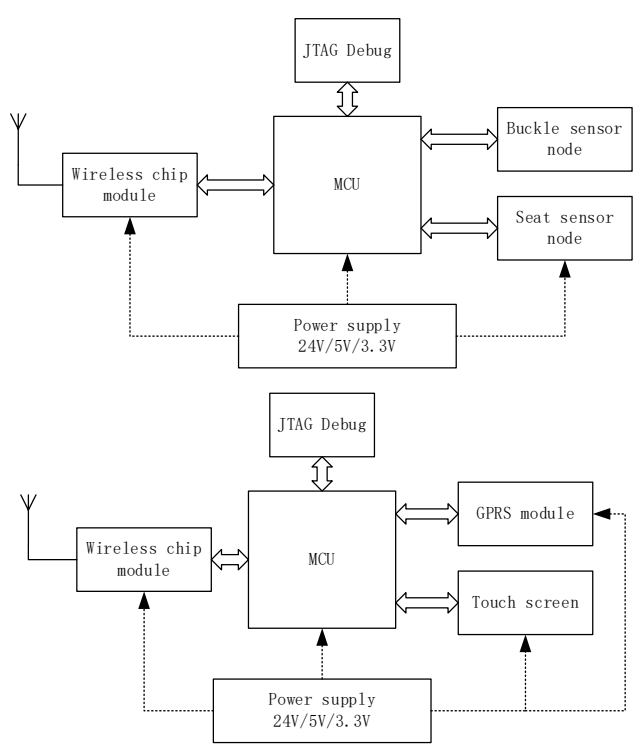

Fig. 3 System hardware components

A detection imaging chip is MC33794, it is a practical circuit, the electric field induction integrated circuit. The chip has nine electrodes and two reference points to test electric field change, 5V operating voltage, the proportion of adjustment and internal key measurement node, through the resistance of the external adjustment precision of sine wave and low cost of the monitoring system and the integrity of the electric field [4].

As shown in Fig. 4, when the body is in the seat of electric field, human equivalent to the external load, then sine wave generator and the human body (external load resistance) cooperate to produce $120 \mathrm{kHz}$ of pure sine wave, different obstacles in the electric field caused changes occurred in an electric field and the corresponding capacitance value is changed, proportional to the measured voltage and $1 / \mathrm{C}$, namely capacitance increase in detection of voltage decreases, so that you can discriminant into the nature of the obstacles in the field, to achieve the purpose of this topic. The detector and the low pass filter are all in the MC33794 chip, and only need to be connected with the capacitance of the $10 \mathrm{nF}$.

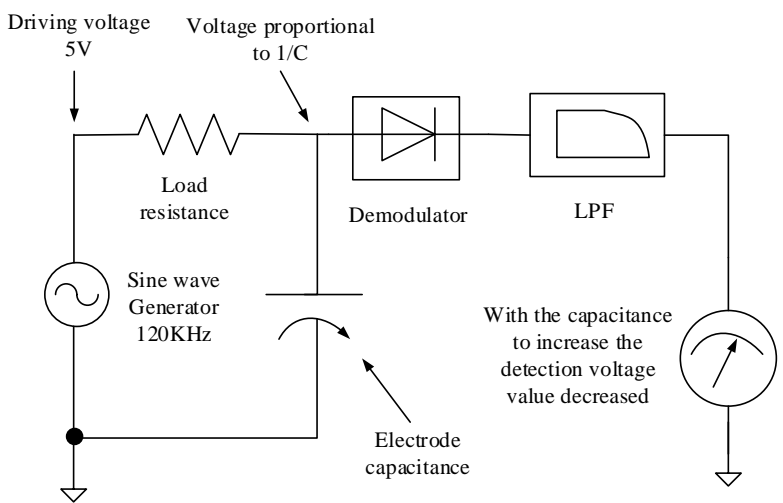

Fig. 4 MC33794

The seating arrangement of the sensor electrode arrangement is shown in Fig. 5. Electric field sensor has a good ability of anti electromagnetic interference and the quick speed of response to transient electric field measurement system can be used to detect the passenger's size and position. Among them, T1, T2, T3, ..., T10 for the emitter, R for the public acceptance. The system uses the public acceptance pole $\mathrm{R}$ and the capacitance change between each emitter to obtain the relevant parameters, which determine the electric field is occupied. Each receiving emitter is placed under the seat, and each pole piece is connected with an electrically conductive sheet. There is an insulating layer between the conductive sheet and the receiving electrode, the insulating layer can shield the electric field line, and avoid the excessive capacitance [5]. 


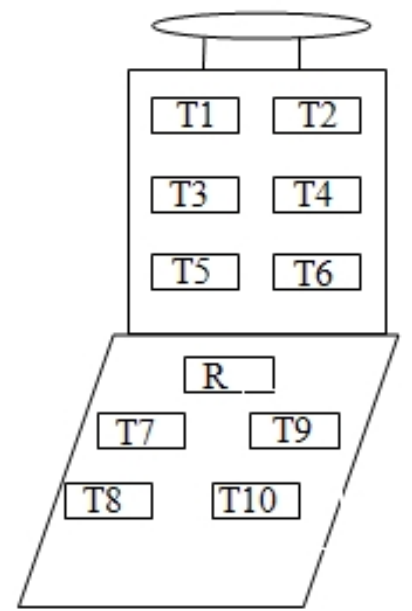

Fig. 5 Seat Electrode Placement

Passenger vehicle central controller of wireless transceiver module of RF chip uses the silicon labs launches integrated degree high, low power, multi band ezradiopro wireless transceiver chip Si4432, its maximum transmission power to 20dbm (100MW), but the receiver sensitivity can -121dbm. Due to the launch of high power, high receiving sensitivity, can be transmitted to thousands of kilometers.

Wireless transceiver transparent module circuit as shown in Fig. 6, SDO, SDI, Si4432, SCLK, nSEL and nIRQ pin is the standard SPI interface, nIRQ is the interrupt output pin. When the overflow data and effective data packet sent or received, CRC errors, detected leading bits and a sync word, power on reset condition occurs and the corresponding interrupt is enabled, the foot of the nIRQ will produces a low level to inform the main control chip stm8s103 to generate an interrupt. SDN foot determines the working state of the Si4432 chip, when the SDN ground (SDN=0), the chip is in the conventional mode of operation; SDN=1, the chip is in power down mode. In power down mode, the contents of the register will be lost, and the SPI is not allowed to be accessed, but the current loss of the chip is only $10 \mathrm{nA}$, and the power consumption is very low.

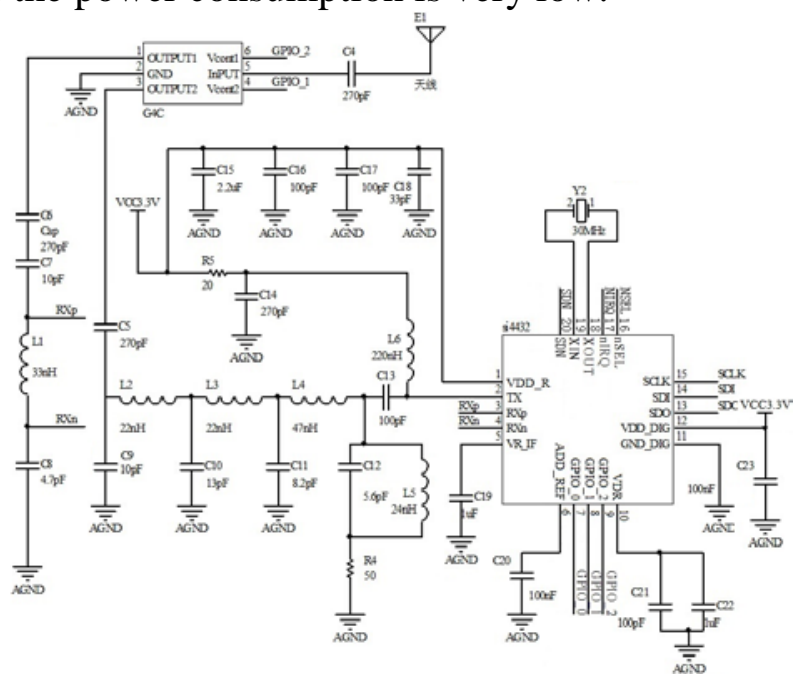

Fig. 6 Wireless Transceiver Circuit

Among them, after connecting to the power supply, the SDN down along the power up and reset, according to the instructions to switch to other work mode. In order to achieve a better communication effect, Si4432 receiver low noise amplifier matching circuit and the power amplifier matching circuit resistance capacitance parameters, here in strict accordance with the data manual provided by the parameter selection. Front end of the diversity circuit using G4C, its Vcont1 feet and Vcont2 feet are connected to the GPIO1 Si4432 and GPIO2. The automatic switching of the transmission and the receiving channel of the cross switch diversity antenna can be realized by this way. 


\section{Monitoring Terminal}

Passenger transportation center monitoring client includes: current all running passenger car safety belt status query, real-time monitoring; when needs to query the status of a route passenger car safety belt queries, i.e., line inquiry; to past one day or a month running safety of vehicles with the history of the state query, a day or a month of support, namely historical query; deletion of vehicle operation, namely the vehicle management; client managers login and password authorization management, the user management; day report printing, namely derived statements; client using the methodology and illustrate, helping.

This part is the main use of the system, the function must be clear. The main display interface, vehicle information, the car seat belt state (four forms). Which left for location information, right above the basic information of the vehicle, the lower right corner selected vehicle safety belt wearing: which when no one seat, safety belt icon display is black; when the error for wear, icons for yellow; when the danger model, the icon is displayed as red; when the safe mode to wear, the icon is displayed for the green. As shown in Fig. 7.

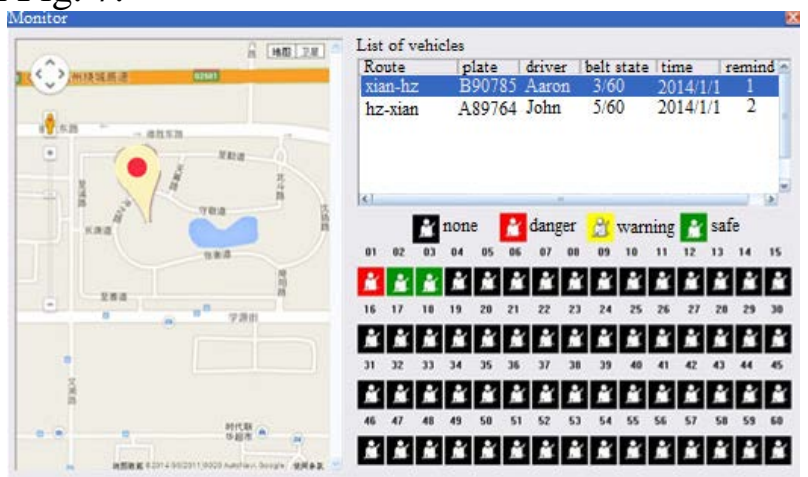

Fig. 7 Monitoring Terminal

\section{Summary}

This paper from the existing passenger car safety belt reminding device technology of, put forward a based on IOT of passenger car safety belt reminder system, it not only makes up the conventional safety belt reminding device just to remind the driver and the copilot and the lack of, also effectively avoids to drawbacks of the video monitoring and propaganda, strengthen the passenger car seat belt wearing management, reduce the workload of passenger transportation management center, to the passenger transportation center can be easily that situation of running passenger car seat belt wearing, can timely remind passengers to wear seat belts, to avoid in the accident because of safety belts did not cause the wear of the casualties.

\section{References}

[1]. Wells J A K, Williams A F, Farmer C M. Seat belt use among African Americans, Hispanics, and whites[J]. Accident Analysis \& Prevention, 2002, 34(4): 523-529.

[2]. Derrig R A, Segui-Gomez M, Abtahi A, et al. The effect of population safety belt usage rates on motor vehicle-related fatalities[J]. Accident Analysis \& Prevention, 2002, 34(1): 101-110.

[3]. Houston D J, Richardson Jr L E. Traffic safety and the switch to a primary seat belt law: the California experience[J]. Accident Analysis \& Prevention, 2002, 34(6): 743-751.

[4]. Cotter W D. Seat belt status external monitoring apparatus and method: U.S. Patent 7,812,716[P]. 2010-10-12.

[5]. George B, Zangl H, Bretterklieber T, et al. Seat occupancy detection based on capacitive sensing[J]. Instrumentation and Measurement, IEEE Transactions on, 2009, 58(5): 1487-1494. 